\title{
Graphene oxide-embedded porous carbon nanofiber webs by electrospinning for capacitive deionization
}

Yu Bai ${ }^{\mathrm{a}}$, Zheng-Hong Huang ${ }^{\mathrm{a},{ }^{*}}$, Xiao-Liang Yu ${ }^{\mathrm{a}}$, Feiyu Kang ${ }^{\mathrm{a}, \mathrm{b}, *}$

${ }^{a}$ Key Laboratory of Advanced Materials (MOE), School of Materials Science and Engineering, Tsinghua University, Beijing 100084, China

${ }^{\mathrm{b}}$ Graduate School at Shenzhen, Tsinghua University, Shenzhen 518055, China

* Corresponding authors. Address: Key Laboratory of Advanced Materials (MOE),

School of Materials Science and Engineering, Tsinghua University, Beijing 100084, China.

Zheng-Hong Huang

Tel: +8610 62773752, Fax: +861062773752.

E-mail address: zhhuang@mail.tsinghua.edu.cn (Z.-H. Huang),

Feiyu Kang

Tel: +861062773752, Fax: +861062773752.

E-mail address: fykang@ @tsinghua.edu.cn (F. Kang).

(C) 2013. This manuscript version is made available under the Elsevier user license http://www.elsevier.com/open-access/userlicense/1.0/ 


\section{Abstract}

Novel freestanding graphene oxide (GO)-embedded porous carbon nanofiber (PCNF) webs were prepared by electrospinning from polyacrylonitrile and GO, followed by carbonization and steam activation, and their electrosorptive performance as binder-free electrodes in capacitive deionization was evaluated. Both GO-PCNF and PCNF demonstrated higher electrosorptive capacities than commercial activated carbon fiber by virtue of smaller diameter and shallower pores. More importantly, GO-PCNF webs processed higher mesopore ratio and electrical conductivity due to GO embedment, which could lead to reduced ion-transport obstacle and enhanced electrical double-layer capacitance, compared to pristine PCNF. Thus, GO-PCNF electrode exhibited better deionization performance.

Key words: Electrospinning; porous carbon nanofiber; graphene oxide; capacitive deionization 


\section{Introduction}

Severe fresh water shortage has occurred globally due to environment contamination and consumption. Capacitive deionization (CDI) which removes ions from brackish water and produces fresh water with less energy and environmental hazard has attracted more and more interests in the past few years [1, 2]. To achieve a high electrosorption capacity for CDI, electrode materials with large surface area and proper functions are the key point [3]. Among various porous carbons with different textures, porous carbon nanofiber (PCNF) webs prepared by electrospinning and subsequent thermal treatment are found to be promising electrode material for CDI process due to freestanding monolithic characteristic and developed pore structure [4-6]. Electrospinning is an effective method to prepare macroscopic fiber webs productively and conveniently [7, 8]. The electrospun PCNF webs can be used directly as electrodes without adding binder and exhibit good ion-removal performance.

Modifying the porous structure and electrical properties of electrospun PCNF webs would further enhance desalination capacity. This optimization can be achieved by adding foreign materials (carbon black and carbon nanotube, etc.) into polymer matrix, which has been demonstrated by the improved physicochemical characteristics of electrospun composite webs in studies for various electrochemical applications [9-11]. Graphene oxide (GO) possesses excellent 2D nanostructure, and its abundant oxygen functional groups can lead to the formation of strong interaction between GO and polymer which is beneficial for the structural stability of webs 
[12-15]. Moreover, reduced graphene oxide (RGO) obtained from GO by thermal treatment possesses higher degree of graphitic structure and consequently outstanding electrical properties $[16,17]$. Thus, GO can serve as good nanofiller to enhance the network structure and electrical properties of PCNF webs.

In the present work, novel GO-embedded PCNF webs were prepared by electrospinning and following thermal treatment, and used directly as CDI electrodes. The resultant webs with GO incorporation possess nanometer-scaled fiber diameter and improved hierarchical pore structure and electrical conductivity, and consequently show higher salt-removal capacity than pristine PCNF webs and commercial activated carbon fiber (ACF).

\section{Experimental}

\subsection{Materials}

Polyacrylonitrile $(\mathrm{PAN}, \mathrm{Mw}=150,000)$ was purchased from Aldrich Chemical Company Inc. (USA). Other reagents were purchased from Beijing Modern Eastern Finechemical Co., Ltd., China. All chemicals were used as received without further purification. For comparison, commercial activated PAN-based carbon fiber web, which was supplied by Challenge Carbon Technology Co., LTD, Taiwan, was used as an electrode material.

\subsection{Synthesis of GO/PCNF webs}

GO nanosheets were prepared from expanded graphite powder according to a modified Hummer's method [18]. (See details in the Supporting Information.) GO nanosheets were dispersed in N, N-dimethylformamide (DMF) and ultrasonically 
treated for 2 h. Then, PAN (10 wt.\%) was added into the GO/DMF solution and stirred at $65^{\circ} \mathrm{C}$ to obtain a homogeneous solution. The weight ratio between PAN and GO was 1:0.05.

The obtained solution was electrospun into GO/PAN nanofiber webs. The electrospinning equipment and process were described in our previous work [19]. The spinning distance between the tip and the collector was $19 \mathrm{~cm}$, the applied voltage was $25 \mathrm{kV}$, and the flow rate of solution was $1 \mathrm{~mL} \mathrm{~h}^{-1}$. Stabilization of the electrospun fibers was carried out by heating to $280{ }^{\circ} \mathrm{C}\left(1{ }^{\circ} \mathrm{C} \min ^{-1}\right)$ in air and maintaining them for $2 \mathrm{~h}$. Finally, the fibers were heated to $800{ }^{\circ} \mathrm{C}\left(5^{\circ} \mathrm{C} \mathrm{min}-1\right)$ in nitrogen and activated by supplying 30 vol.\% steam for $30 \mathrm{~min}$. The obtained $\mathrm{GO} /$ porous carbon nanofiber web was denoted as GO-PCNF. For comparison, pristine porous carbon nanofiber web was fabricated, denoted as PCNF.

\subsection{Characterization}

The scanning electron microscope (SEM, LEO-1530, Germany) and Raman spectroscopy (Renishaw Invia RM200, England) were used to study the surface morphology and structure of webs. Pore structural properties were investigated from $\mathrm{N}_{2}$ adsorption at $77 \mathrm{~K}$ (Quantachrome Autosorb-1, USA). The samples were degassed at $195{ }^{\circ} \mathrm{C}$ for about $18 \mathrm{~h}$ before the test. Galvanostatic constant current charging/discharging tests of as-made webs were carried out on an Arbin-BT2000 (Arbin Instruments) workstation using a three-electrode testing system with a platinum foil as counter electrode and a $\mathrm{Hg} / \mathrm{HgO}$ reference electrode in $6 \mathrm{M} \mathrm{KOH}$ aqueous electrolyte. 


\subsection{CDI measurements}

The various webs were tested as CDI electrodes in an electrosorptive setup which was illustrated in our previous work [4]. $\mathrm{NaCl}$ solution was pumped into the bottom and extracted from the top of the cell by a peristaltic pump with a flow rate of $6 \mathrm{~mL}$ $\min ^{-1}$. In all experiments the operation voltage was $1.2 \mathrm{~V}$, and the volume of $\mathrm{NaCl}$ solution was $9 \mathrm{~mL}$. The solution conductivity was continuously monitored at $10 \mathrm{~s}$ intervals using a conductivity meter (type 308A, Leici Company). In the regeneration process, the operation voltage was canceled, while $\mathrm{NaCl}$ solution was still pumped continuously. When the concentration returned to the initial value, the next deionization process started.

The electroadsorption capacity $Q_{t}$ can be calculated according to the following equation:

$$
Q_{t}=\left(C_{0}-C_{t}\right) \times V_{\mathrm{NaCl}} / M
$$

Here $C_{0}\left(\mathrm{mg} \mathrm{L}^{-1}\right)$ is the initial concentration of $\mathrm{NaCl}, C_{t}$ is the instantaneous concentration of $\mathrm{NaCl}$ at time $t, V_{\mathrm{NaCl}}(\mathrm{mL})$ is the solution volume, and $M(\mathrm{~g})$ is the mass of various web electrodes.

\section{Results and discussion}

\subsection{Materials characterization}



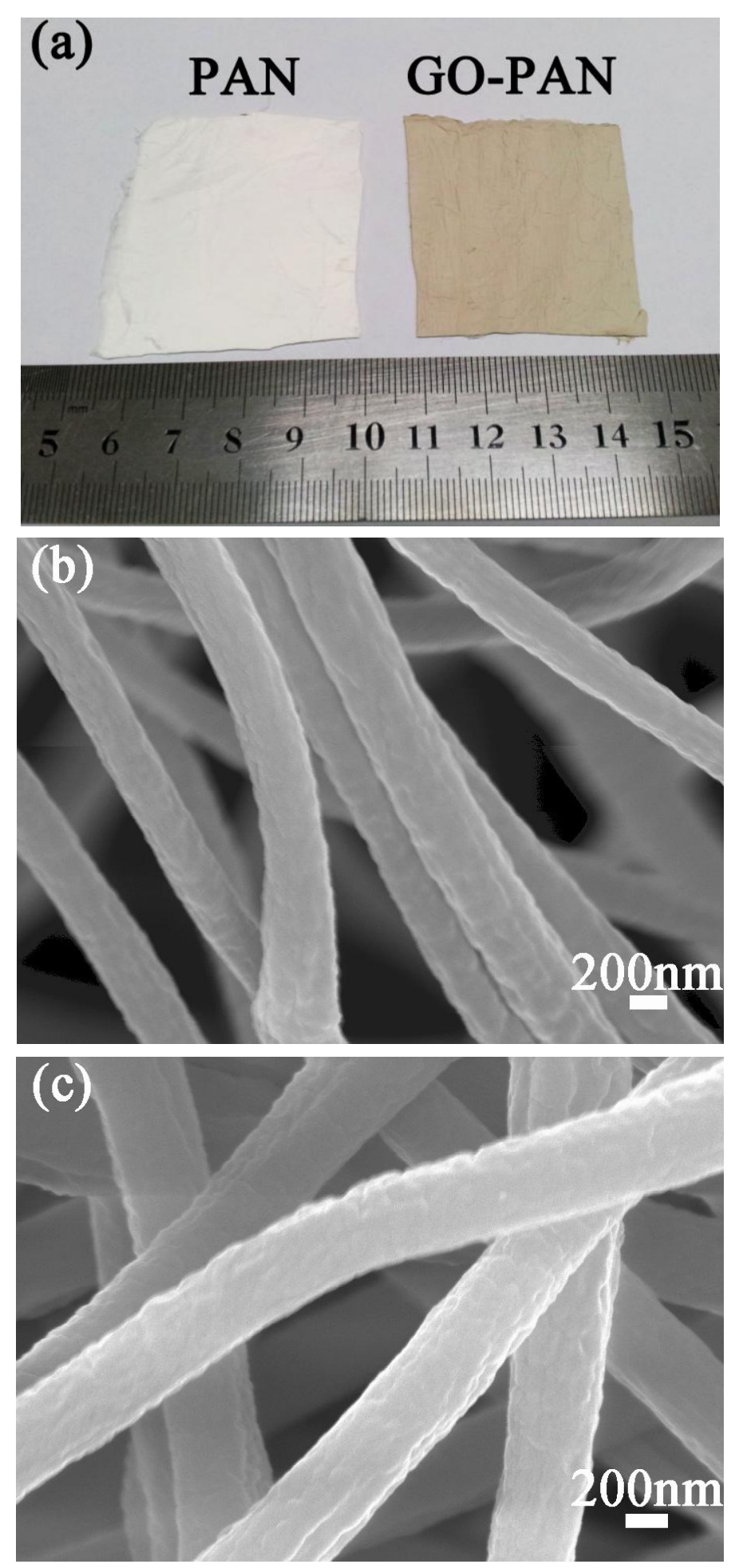

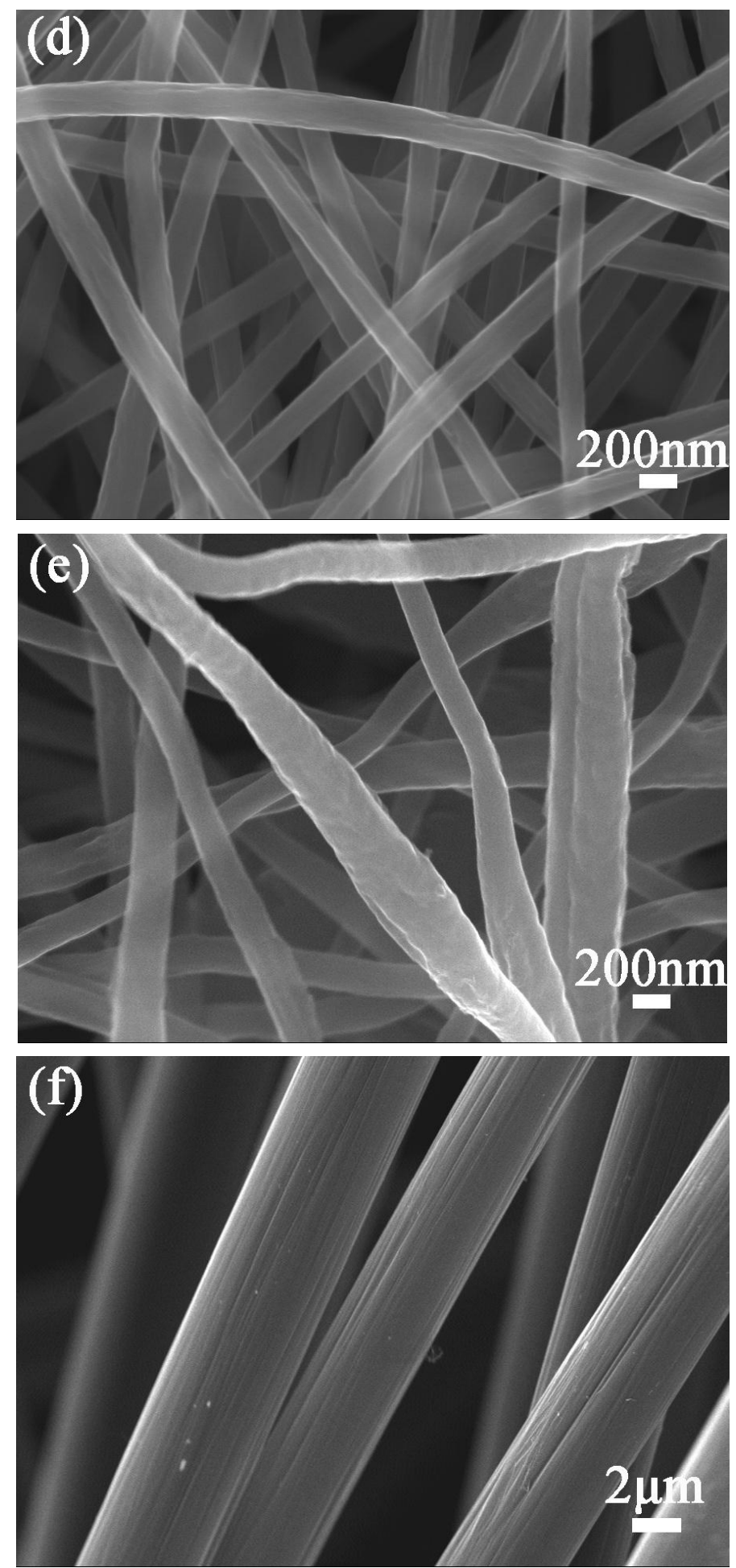

Fig. 1. (a) Digital image of as-spun nanofiber webs. SEM images of (b) PAN, (c) GO-PAN, (d) PCNF, (e) GO-PCNF and (f) ACF.

The digital image of as-spun webs is shown in Fig. 1a. Electrospun webs exhibit 
freestanding characteristic, and color changes from white to grey with GO embedding, suggesting that GO nanosheets have been dispersed in the PAN substrate. According to Fig. S1 in the Supporting Information, both PCNF and GO-PCNF are black freestanding carbon webs. SEM images show that as-spun and carbon nanofiber webs possess consecutive fibrous morphology and interwoven network structure, and diameters of carbon nanofibers reduced due to burn off during carbonization and activation. It can be seen that obvious nonuniformity and roughness exist in GOembedded nanofibers which have slightly larger diameter than pristine nanofibers owing to increased viscosity of spinning solution. No GO aggregate can be seen for GO-PAN and GO-PCNF. In addition, the fiber diameter of commercial ACF is around $5 \mu \mathrm{m}$ (Fig. 1f), much larger than the cases of PCNF and GO-PCNF.

Raman spectra in Fig. 2 show that as-spun GO-PAN web exhibits two additional peaks at around 1350 ( $\mathrm{D}$ band, the defects and disordered band) and 1590 ( $\mathrm{G}$ band, the ordered graphitic band) $\mathrm{cm}^{-1}$ in comparison to pristine PAN web, demonstrating that GO has been embedded into the PAN substrate. After thermal treatment, both PCNF and GO-PCNF exhibit overlapping D band and G band. The intensity ratios of $\mathrm{D}$ band to $\mathrm{G}$ band $\left(I_{\mathrm{D}} / I_{\mathrm{G}}\right)$ for PCNF and GO-PCNF are 3.56 and 3.15, respectively, indicating that GO-PCNF web has higher degree of graphitic structure than pristine web [10]. This can be attributed to the fact that GO is partly transformed to RGO with relatively ordered structure by thermal treatment, considering that thermal annealing is an effective reduction strategy to obtain RGO from GO $[16,20]$. Moreover, the existence of GO and RGO can potentially promote 
the formation of ordered graphite carbon during the process of carbonization and activation [17].

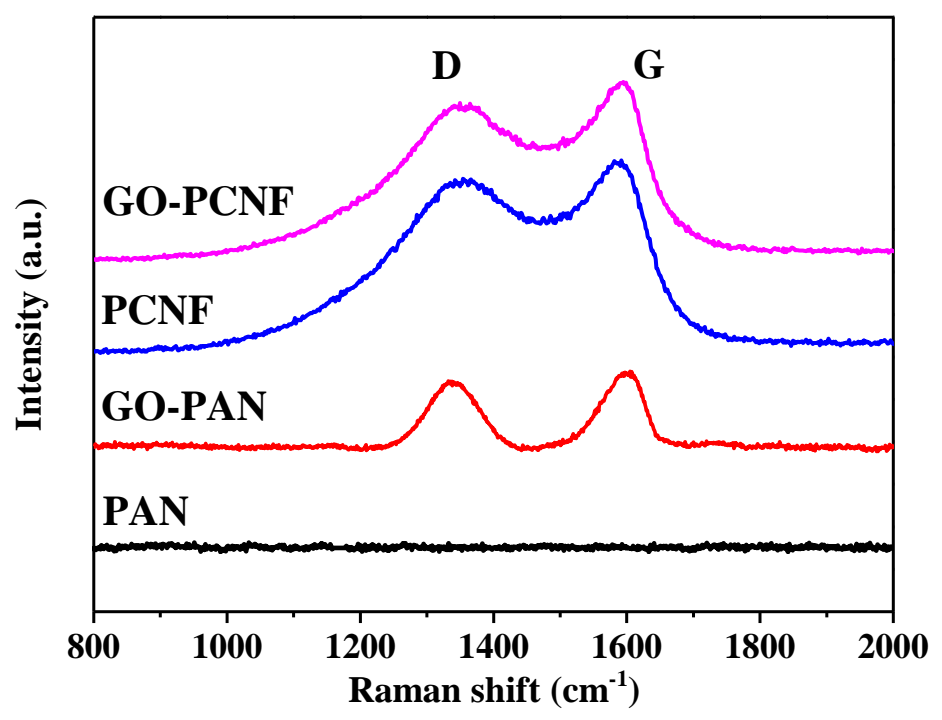

Fig. 2. Raman spectra of various webs.

Fig. 3a shows the $\mathrm{N}_{2}$ adsorption/desorption isotherms at $77 \mathrm{~K}$ of PCNF, GO-PCNF and ACF. The isotherms of PCNF and ACF belong to type I in IUPAC classification, demonstrating that micropores are dominant [21]. While for GO-PCNF, the amounts adsorbed rise steeply close to the saturation vapor pressure, which means unrestricted multilayer formation process of large mesopores and macropores [22]. Pore structural parameters of three webs are listed in Table 1. ACF possesses the highest specific surface area and pore volume. However, the mesopore volume and mesopore ratio of GO-PCNF web are higher than those of other webs. Pore size distributions of three webs calculated by non-local density function theory $[23,24]$ are shown in Fig. 3b. From PCNF to GO-PCNF, pores with widths of 0.5-0.7 nm 
decrease sharply and pore widths shift to larger values, indicating that some micropores have expanded to larger pores, which coincides with the increased mesopores for GO-PCNF (Fig. 3b inset and Table 1). The well-developed hierarchical pore structure in GO-PCNF web could be attributed to the incorporation of GO. Some larger pores are created due to phase separation and different shrinkage between the GO and the PAN during carbonization and activation process [11]. Hierarchical pore structure with higher mesopore ratio will favor the desalination performance of electrodes by minimizing the resistance and diffusion distance of ions for CDI [25, 26].

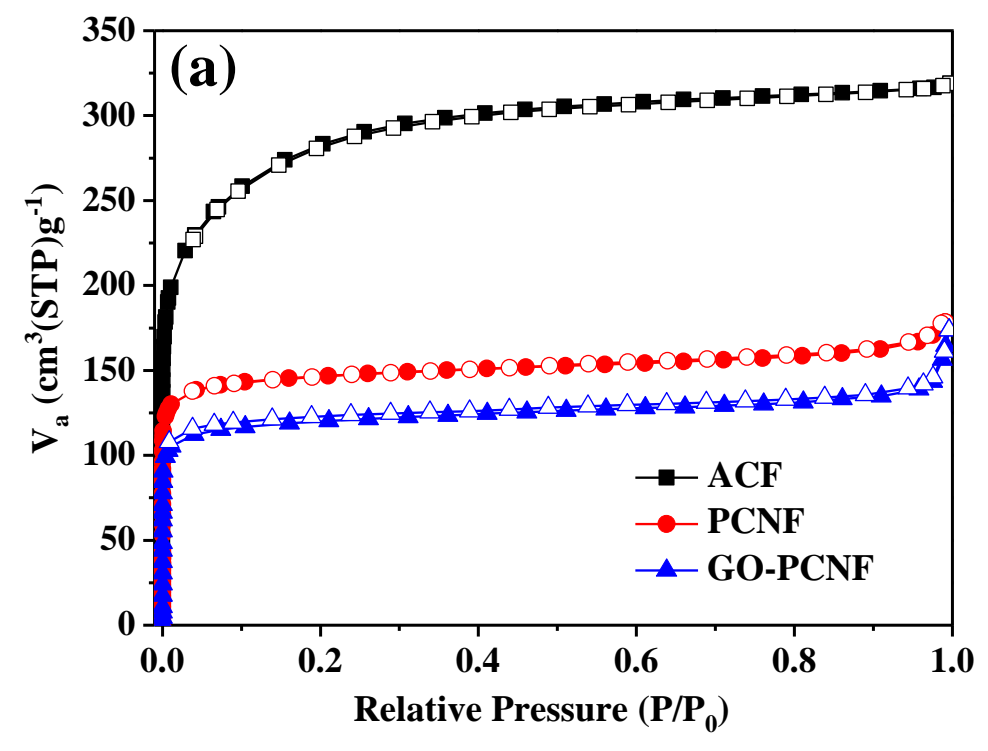




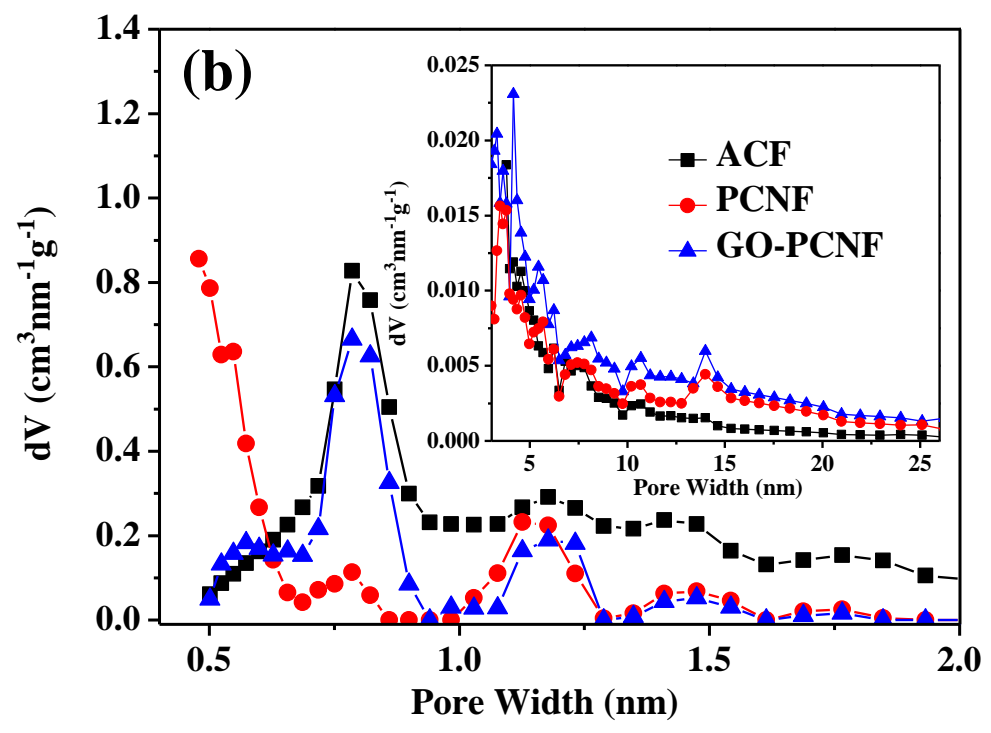

Fig. 3. (a) $\mathrm{N}_{2}$ adsorption/desorption isotherms at $77 \mathrm{~K}$ and (b) micropore and mesopore (inset) size distributions of PCNF, GO-PCNF and ACF.

Table 1 Pore structural parameters of various webs.

\begin{tabular}{lllll}
\hline Sample & $\begin{array}{l}S^{\mathrm{a}} \\
\left(\mathrm{m}^{2} \mathrm{~g}^{-1}\right)\end{array}$ & $\begin{array}{l}V_{\mathrm{T}}{ }^{\mathrm{b}} \\
\left(\mathrm{cm}^{3} \mathrm{~g}^{-1}\right)\end{array}$ & $\begin{array}{l}V_{\text {meso }}{ }^{\mathrm{c}} \\
\left(\mathrm{cm}^{3} \mathrm{~g}^{-1}\right)\end{array}$ & $\begin{array}{l}V_{\text {meso }} / V_{\mathrm{T}} \\
(\%)\end{array}$ \\
\hline PCNF & 583 & 0.273 & 0.053 & 19 \\
GO-PCNF & 474 & 0.259 & 0.097 & 37 \\
ACF & 1047 & 0.493 & 0.051 & 10 \\
\hline
\end{tabular}

${ }^{\text {a }} S$ : specific surface area by $\alpha_{\mathrm{s}}$ method.

${ }^{\mathrm{b}} V_{\mathrm{T}}$ : total pore volume according to the capacity of $\mathrm{N}_{2}$ adsorption isotherm at $\mathrm{P} / \mathrm{P}_{0}$ of 0.99 .

${ }^{\mathrm{c}} V_{\text {meso }}$ : mesopore volume by BJH method.

Fig. 4 shows the charge/discharge curves at a current density of $0.5 \mathrm{~A} \mathrm{~g}^{-1}$ to evaluate the electrochemical performance of PCNF and GO-PCNF as self-standing 
electrodes. The beginning of the discharge curve exhibits a steep drop, i.e., the IR drop caused by the internal resistance of electrodes [17]. The IR drop of GO-PCNF is lower than that of PCNF, implying that smaller contact resistance and internal resistance exist in GO-PCNF electrodes compared to PCNF. Therefore, GO-embedded nanofiber webs have increased electrical conductivity, which is consistent with their higher degree of graphitization (Fig. 2). This is obviously attributed to the existence of partly obtained RGO with advantageous electrical properties in carbon nanofibers. Additionally, the specific capacitance can be calculated from the discharging curve as:

$$
C=I \times \Delta t /(M \times \Delta V)
$$

where $I(\mathrm{~A})$ is the applied current, $\Delta t(\mathrm{~s})$ is the discharge time, $M(\mathrm{~g})$ is the mass of electrode material, and $\Delta V(\mathrm{~V})$ is the discharging potential difference. The specific capacitance of GO-PCNF electrodes is calculated as $151.6 \mathrm{~F} \mathrm{~g}^{-1}$, higher than that of PCNF (105.9 $\left.\mathrm{F} \mathrm{g}^{-1}\right)$. Thus, enhanced electrical double-layer capacitance is observed with GO-PCNF electrodes. The formation of interconnected hierarchical structure due to GO incorporation improves the electrical conductivity of nanofiber webs and allows for outstanding electrochemical deionization performance [27, 28]. 


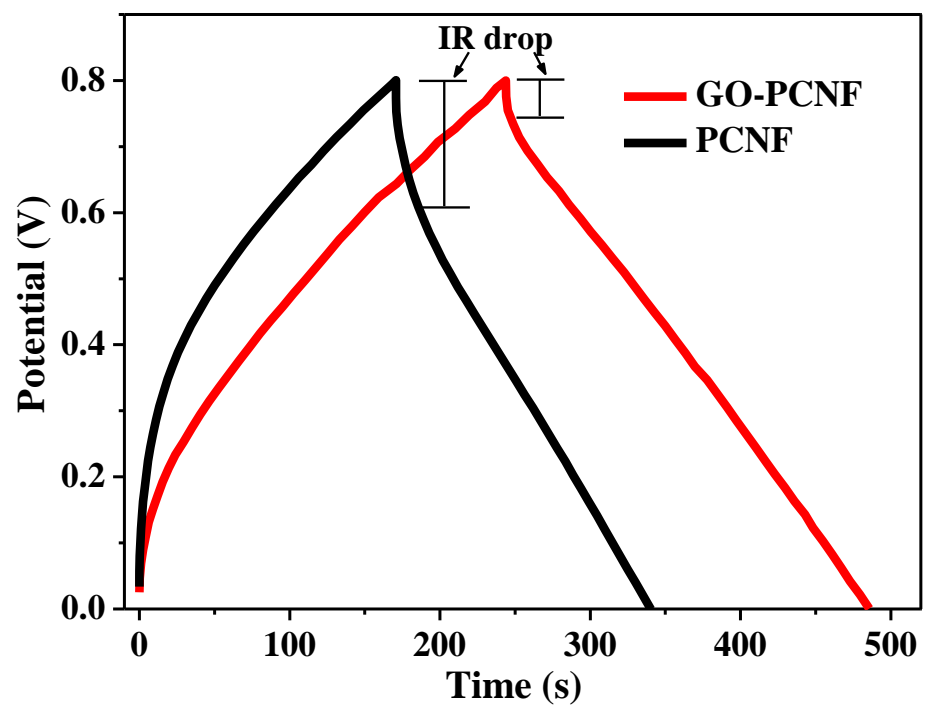

Fig. 4. Galvanostatic charge/discharge curves of PCNF and GO-PCNF webs at a current density of $0.5 \mathrm{~A} \mathrm{~g}^{-1}$.

\subsection{CDI performance}

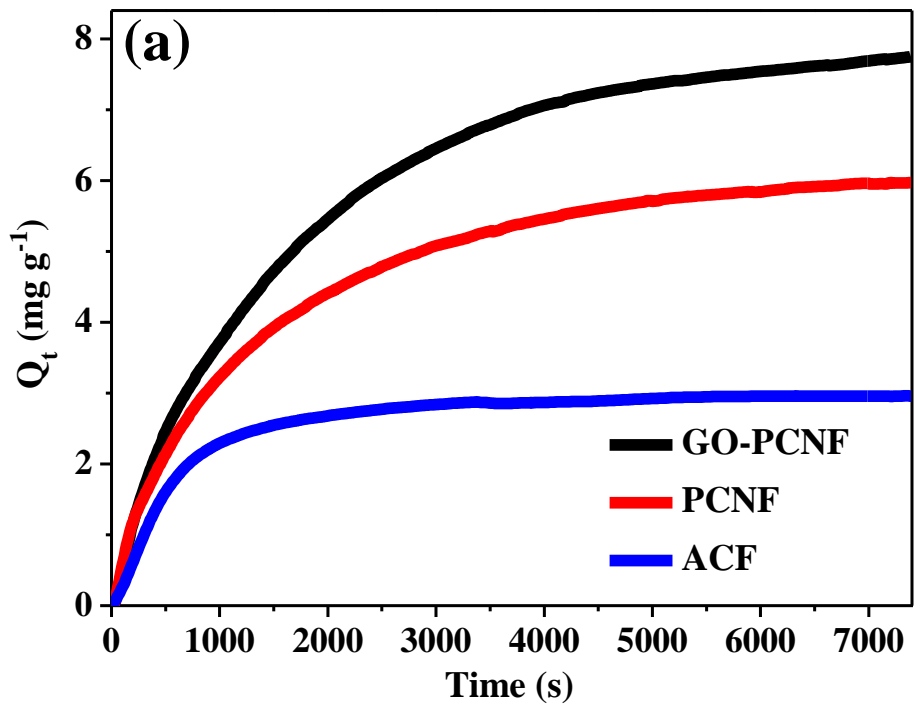



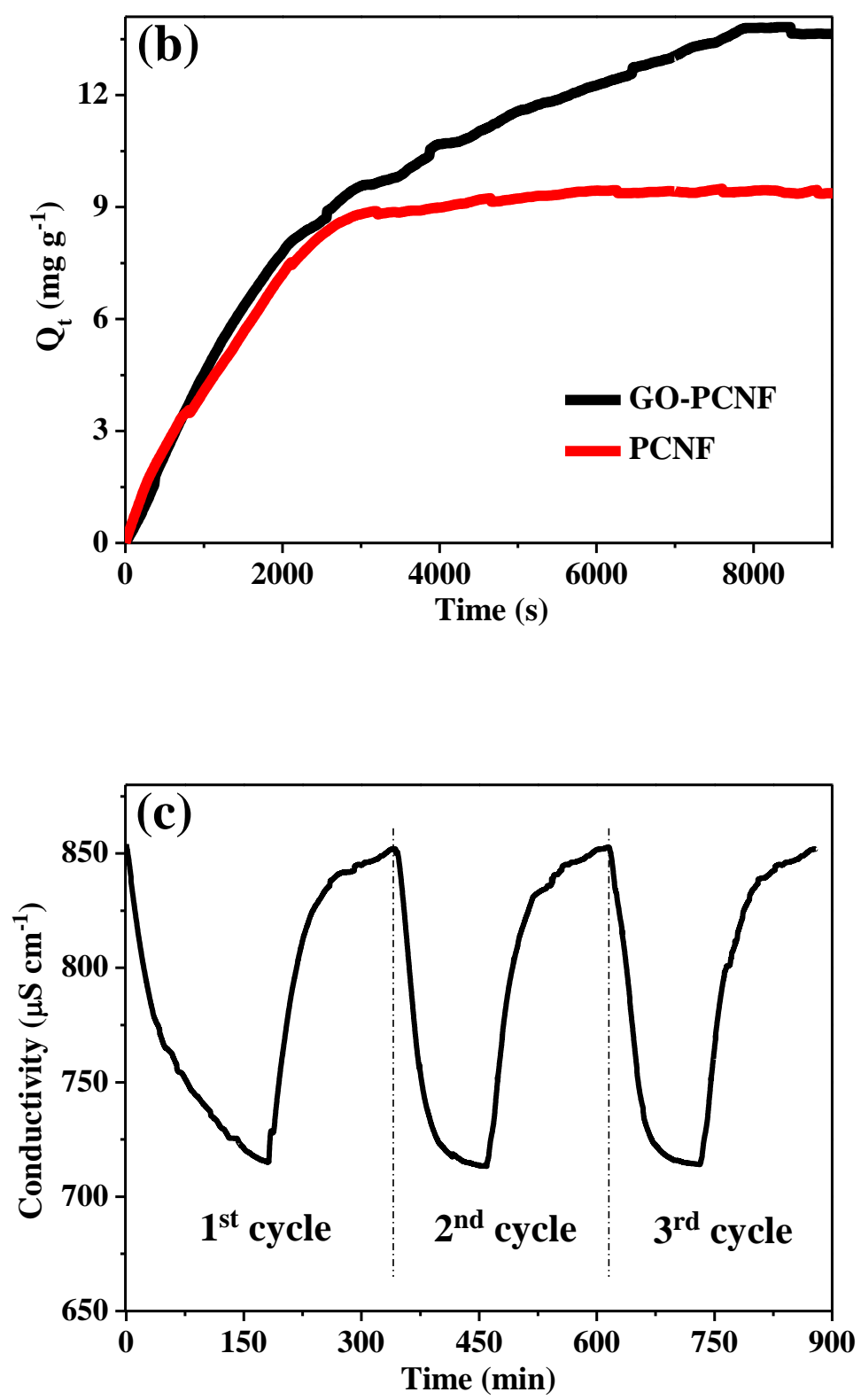

Fig. 5. Electrosorption amount of various web electrodes as a function of time, for which (a) $C_{0}=100 \mathrm{mg} \mathrm{L}^{-1}$, (b) $C_{0}=450 \mathrm{mg} \mathrm{L}^{-1}$. (c) Desalination cycling of GO-PCNF electrode with initial concentration of $450 \mathrm{mg} \mathrm{L}^{-1}$.

The three webs were tested directly as CDI electrodes without the addition of binder. The electrode was firstly washed with pure water until the solution 
conductivity approached zero, and the ion-removal capacity of the webs was recorded. The electrosorption capacities of PCNF, GO-PCNF and ACF webs are shown in Fig. 5. Once the electric field was applied, the adsorption amount increased sharply. Then, the change became gradually lower until equilibrium was reached. It can be noted that both GO-PCNF and PCNF show much higher ion-removal capacities than commercial ACF, even though considerably developed microporous structure is associated with ACF web (Table 1). This result suggests that electrospun webs with nanometer-scaled diameter are more favorable for CDI process than conventional carbon fiber webs (micrometer scale). Carbon nanofibers with much smaller diameter possess shallower pores and more developed interconnected network architecture than conventional carbon fibers $[29,30]$, which dramatically shortens the transportation distances for ions to the interior surface of electrodes and makes more ions go into pores easily, leading to an enhanced capacitive desalination.

Meanwhile, quantity electroadsorbed by GO-PCNF electrode is higher than the case of PCNF electrode (Fig. 5a and b). At $t=120 \mathrm{~min}$, the electrosorption equilibrium is almost reached, and the respective electrosorption capacities of GO-PCNF and PCNF electrodes are 7.8 and $5.9 \mathrm{mg} \mathrm{g}^{-1}\left(C_{0}=100 \mathrm{mg} \mathrm{L}^{-1}\right), 13.2$ and $9.4 \mathrm{mg} \mathrm{g}^{-1}\left(C_{0}=450 \mathrm{mg} \mathrm{L}^{-1}\right)$. The improved mesopore ratio and electrical conductivity resulting from the addition of GO minimize the diffusion resistance and distance of ions and improve the electrical connection for CDI, which is responsible for the enhanced capacitive desalination performance of GO-PCNF.

In addition, cyclic electrosorption and regeneration of GO-PCNF electrode 
were performed to evaluate the reversibility of electrode material in CDI cells. Fig. 5c shows that there is almost no decrease in the electrosorption capacity over three cycles, demonstrating that the desalination process can be repeated very well. Thus, CDI process with GO-PCNF electrode exhibits advantageous reversibility.

\section{Conclusion}

Novel freestanding GO-embedded porous carbon nanofiber webs are successfully fabricated by electrospinning and subsequent carbonization and steam activation. The addition of GO can improve the mesoporosity and the electrical conductivity of nanofiber webs, which is beneficial for the ion-transport for CDI process. The GO-embedded nanofiber webs with nanometer-scaled fiber diameter, enhanced electrical connection and tuned hierarchical structure exhibit higher electrosorption capacity than pristine carbon nanofiber webs and commercial ACF, and provide great promise for use as electrodes for CDI. More technical routes such as optimizing the percent of GO adding are still needed to enhance the desalination performance of nanofiber webs in further study.

\section{Acknowledgements}

The authors acknowledge the financial support of the National Natural Science Foundation of China (Grant No. 51072091) and the cooperative project JST-MOST (No. 2011DFA50430).

\section{Appendix A. Supplementary material}

Method of GO synthesis and digital image of PCNF and GO-PCNF.

\section{References}


[1] Y. Oren, Capacitive delonization (CDI) for desalination and water treatment - past, present and future (a review), Desalination 228 (2008) 10-29.

[2] J.Y. Lee, S.J. Seo, S.H. Yun, S.H. Moon, Preparation of ion exchanger layered electrodes for advanced membrane capacitive deionization (MCDI), Water Res. 45 (2011) 5375-5380.

[3] S. Nadakatti, M. Tendulkar, M. Kadam, Use of mesoporous conductive carbon black to enhance performance of activated carbon electrodes in capacitive deionization technology, Desalination 268 (2011) 182-188.

[4] M. Wang, Z.H. Huang, L. Wang, M.X. Wang, F.Y. Kang, H.Q. Hou, Electrospun ultrafine carbon fiber webs for electrochemical capacitive desalination, New J. Chem. 34 (2010) 1843-1845.

[5] G. Wang, C. Pan, L.P. Wang, Q. Dong, C. Yu, Z.B. Zhao, J.S. Qiu, Activated carbon nanofiber webs made by electrospinning for capacitive deionization, Electrochim. Acta 69 (2012) 65-70.

[6] Y. Bai, Z.-H. Huang, Z.-X. Zhang, F. Kang, Improvement of the hydrophilicity of electrospun porous carbon nanofibers by grafting phenylsulfonic acid groups, J. Colloid Interface Sci. 394 (2013) 177-182.

[7] D. Li, Y.N. Xia, Electrospinning of nanofibers: Reinventing the wheel? Adv. Mater. 16 (2004) 1151-1170.

[8] A. Greiner, J.H. Wendorff, Electrospinning: A fascinating method for the preparation of ultrathin fibres, Angew. Chem.-Int. Edit. 46 (2007) 5670-5703.

[9] H.Q. Hou, J.J. Ge, J. Zeng, Q. Li, D.H. Reneker, A. Greiner, S.Z.D. Cheng, 
Electrospun polyacrylonitrile nanofibers containing a high concentration of well-aligned multiwall carbon nanotubes, Chem. Mater. 17 (2005) 967-973.

[10] J.-G. Wang, Y. Yang, Z.-H. Huang, F. Kang, Synthesis and electrochemical performance of $\mathrm{MnO} 2 / \mathrm{CNTs}$-embedded carbon nanofibers nanocomposites for supercapacitors, Electrochim. Acta 75 (2012) 213-219.

[11] G. Wang, Q. Dong, Z. Ling, C. Pan, C. Yu, J.S. Qiu, Hierarchical activated carbon nanofiber webs with tuned structure fabricated by electrospinning for capacitive deionization, J. Mater. Chem. 22 (2012) 21819-21823.

[12] H.D. Huang, P.G. Ren, J. Chen, W.Q. Zhang, X. Ji, Z.M. Li, High barrier graphene oxide nanosheet/poly(vinyl alcohol) nanocomposite films, J. Membr. Sci. 409 (2012) 156-163.

[13] A.K. Geim, Graphene: Status and Prospects, Science 324 (2009) 1530-1534.

[14] X.M. Yang, Y.F. Tu, L.A. Li, S.M. Shang, X.M. Tao, Well-Dispersed Chitosan/Graphene Oxide Nanocomposites, ACS Appl. Mater. Interfaces 2 (2010) 1707-1713.

[15] H.R. Pant, C.H. Park, L.D. Tijing, A. Amarjargal, D.H. Lee, C.S. Kim, Bimodal fiber diameter distributed graphene oxide/nylon-6 composite nanofibrous mats via electrospinning, Colloid Surf. A-Physicochem. Eng. Asp. 407 (2012) $121-125$.

[16] S.F. Pei, H.M. Cheng, The reduction of graphene oxide, Carbon 50 (2012) $3210-3228$.

[17] Z.P. Zhou, X.F. Wu, Graphene-beaded carbon nanofibers for use in 
supercapacitor electrodes: Synthesis and electrochemical characterization, J. Power Sources 222 (2013) 410-416.

[18] W.S. Hummers, R.E. Offeman, Preparation of graphitic oxide, J. Am. Chem. Soc. 80 (1958) 1339-1339.

[19] M.-X. Wang, Z.-H. Huang, F. Kang, K. Liang, Porous carbon nanofibers with narrow pore size distribution from electrospun phenolic resins, Mater. Lett. 65 (2011) 1875-1877.

[20] H.C. Schniepp, J.L. Li, M.J. McAllister, H. Sai, M. Herrera-Alonso, D.H. Adamson, R.K. Prud'homme, R. Car, D.A. Saville, I.A. Aksay, Functionalized single graphene sheets derived from splitting graphite oxide, J. Phys. Chem. B 110 (2006) 8535-8539.

[21] S. Brunauer, L.S. Deming, W.E. Deming, E. Teller, On a theory of the van der Waals adsorption of gases, J. Am. Chem. Soc. 62 (1940) 1723-1732.

[22] W.F. Zhang, Z.H. Huang, G.P. Cao, F.Y. Kang, Y.S. Yang, A novel mesoporous carbon with straight tunnel-like pore structure for high rate electrochemical capacitors, J. Power Sources 204 (2012) 230-235.

[23] C. Lastoskie, K.E. Gubbins, N. Quirke, Pore-size distribution analysis of microporous carbons - a density-functional theory approach, J. Phys. Chem. 97 (1993) 4786-4796.

[24] P. Tarazona, U.M.B. Marconi, R. Evans, Phase-equilibria of fluid interfaces and confined fluids - nonlocal versus local density functionals, Mol. Phys. 60 (1987) 573-595. 
[25] G. Hasegawa, M. Aoki, K. Kanamori, K. Nakanishi, T. Hanada, K. Tadanaga, Monolithic electrode for electric double-layer capacitors based on macro/meso/microporous S-Containing activated carbon with high surface area, J. Mater. Chem. 21 (2011) 2060-2063.

[26] P. Adelhelm, Y.S. Hu, L. Chuenchom, M. Antonietti, B.M. Smarsly, J. Maier, Generation of hierarchical meso- and macroporous carbon from mesophase pitch by spinodal decomposition using polymer templates, Adv. Mater. 19 (2007) 4012-4017.

[27] C.Y. Nie, L.K. Pan, H.B. Li, T.Q. Chen, T. Lu, Z. Sun, Electrophoretic deposition of carbon nanotubes film electrodes for capacitive deionization, J. Electroanal. Chem. 666 (2012) 85-88.

[28] Y.K. Zhan, C.Y. Nie, H.B. Li, L.K. Pan, Z. Sun, Enhancement of electrosorption capacity of activated carbon fibers by grafting with carbon nanofibers, Electrochim. Acta 56 (2011) 3164-3169.

[29] K.J. Lee, N. Shiratori, G.H. Lee, J. Miyawaki, I. Mochida, S.-H. Yoon, J. Jang, Activated carbon nanofiber produced from electrospun polyacrylonitrile nanofiber as a highly efficient formaldehyde adsorbent, Carbon 48 (2010) $4248-4255$.

[30] C. Kim, B.T.N. Ngoc, K.S. Yang, M. Kojima, Y.A. Kim, Y.J. Kim, M. Endo, S.C. Yang, Self-sustained thin webs consisting of porous carbon nanofibers for supercapacitors via the electrospinning of polyacrylonitrile solutions containing zinc chloride, Adv. Mater. 19 (2007) 2341-2346. 
Graphical Abstract

\section{Graphical Abstract}

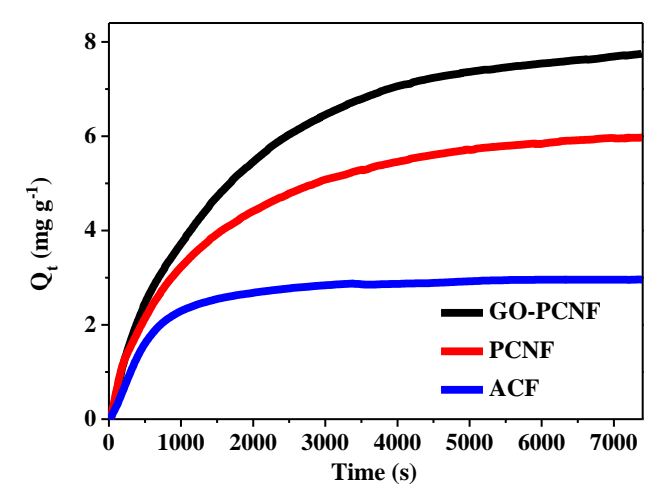

